RUNNING HEAD: FUNCTIONAL-COGNITIVE FRAMEWORK

Why the cognitive approach in psychology would profit from a functional approach and vice versa

Jan De Houwer

Ghent University, Ghent, Belgium

In Press. Perspectives on Psychological Science.

mailing address:

Jan De Houwer

Ghent University

Henri Dunantlaan 2

B-9000 Ghent

Belgium

email: Jan.DeHouwer@UGent.be

phone: 003292646445

fax: 003292646489 


\begin{abstract}
Cognitively oriented psychologists often define behavioral effects in terms of mental constructs (e.g., classical conditioning as a change in behavior that is due to the formation of associations in memory) and thus effectively treat those effects as proxies for mental constructs. This practice can, however, hamper scientific progress. I argue that if psychologists would consistently define behavioral effects only in terms of the causal impact of elements in the environment (e.g., classical conditioning as a change in behavior that is due to the pairing of stimuli), they would de facto adopt a functional approach that not only reveals the environmental causes of behavior but also optimizes cognitive research. The cognitive approach in turn strengthens the functional approach by facilitating the discovery of new causal relations between the environment and behavior. I thus propose a functionalcognitive framework for research in psychology that capitalizes on the mutually supportive nature of the functional and cognitive approaches in psychology.
\end{abstract}




\section{Why the cognitive approach in psychology would profit from a functional approach and vice versa}

The cognitive approach in psychology has dominated psychological research for almost half of a century now. Cognitively oriented researchers aim to understand the nature of the mental processes and mental representations by which organisms store, process, and retrieve information and that are assumed to guide behavior (e.g., Neiser, 1967). Despite many remarkable successes (see Lamberts \& Goldstone, 2004, for reviews), the cognitive approach also has limitations. Most importantly, many mental constructs cannot be observed directly. Researchers often try to overcome this problem by using directly observable behavior as a proxy for mental constructs. In the first part of this paper, I argue that the use of proxies can hamper research and theorizing. Next, I put forward a functional-cognitive framework in which the cognitive approach is strengthened by combining it with a functional approach aimed at revealing the environmental causes of behavior. Then, I consider potential arguments against combining the functional and cognitive approaches. Finally, I list a number of recommendations for psychological research

\section{Limitations of the Cognitive Approach in Psychology}

\section{Using behavioral effects as proxies for mental constructs}

Mental constructs refer to a realm outside of the physical world in which information is represented and processed independently of the physical system in which it is implemented (e.g., the brain, a computer; see Brysbaert \& Rastle, 2009; Gardner, 1987). Many mental processes and representations cannot be observed directly but can only be inferred from how a system (e.g., a human, a computer) responds to certain situations. For instance, whereas the mental representation of a chair cannot be observed directly, one can infer from how someone reacts to a chair (e.g., sits on it) whether that person possesses certain information 
about chairs.

The fact that many mental representations and mental processes cannot be observed directly of course complicates the study of those constructs. One way to circumvent these problems is to use behavioral effects (i.e., patterns of behaviors in certain situations) as proxies for mental constructs. These effects are assumed to function as a substitute for the mental constructs in that their presence can be treated as equivalent to the presence of the mental constructs themselves. Therefore, the effects can be defined in terms of mental constructs and the conditions under which they occur provide a direct reflection of the properties of the mental constructs. For example, cognitive learning researchers who study

the mental process of association formation in memory (see Bouton, 2007, for a review) often use the presence of classical conditioning effects as a proxy for the formation of associations in memory. Whenever classical conditioning is observed (e.g., a dog starts salivating to a tone after that tone has been paired with food), they conclude that association formation has taken place. Likewise, researchers have used negative priming effects (i.e., the observation that responses to a stimulus are slowed down when the stimulus is preceded by a related stimulus) as a proxy for the mental process of the inhibition of mental representations (e.g., Joormann, 2004; Tipper, 1985).

\section{Problems with using behavioral effects as proxies of mental constructs}

Unfortunately, using behavioral effects as a proxy for mental constructs is problematic in several respects. These problems arise from the fact that it implies a priori assumptions about the relation between mental constructs and behavior. As such, using behavioral effects as a proxy for mental constructs violates the general scientific principle that the explanandum (that which needs to be explained; in this case behavioral effects) needs to be kept separate from the explanans (that which is used to explain; in this case mental 
constructs; Hempel, 1970). For a behavioral effect to be a proxy of a mental construct, it does not suffice that the effect can be explained on the basis of the mental construct (e.g., that association formation can produce classical conditioning or that the inhibition of representations can result in negative priming). In order to make the reverse inference from the presence of the effect to the presence of the mental construct, the mental construct needs to be a necessary condition for the effect (i.e., the effect can be present only if the mental construct is present). If it turns out that the behavioral effect can be caused by other mental constructs in the absence of the to-be-registered mental construct, then the presence of the behavioral effect does not allow one to draw firm conclusions about the to-be-registered mental construct (also see Borsboom, Mellenbergh, \& van Heerden, 2004; Poldrack, 2006). For instance, the negative priming effect has been largely abandoned as a proxy for inhibition because it has been argued that the effect can be caused by mental processes other than inhibition (e.g., Neill \& Valdes, 1992). Similarly, evidence supporting the idea that conditioning effects are not necessarily due to the formation of associations in memory (e.g., Mitchell, De Houwer, \& Lovibond, 2009) raises doubts about whether the presence of conditioning effects demonstrates the presence of association formation.

One could nevertheless continue to use behavioral effects as proxies for mental constructs as long as there are no reasons to doubt the usefulness of those proxies. When doubts are raised about existing behavioral proxies, one can simply switch to new behavioral proxies that are still undisputed. There are, however, at least two problems with such an approach. First, it can lead to a self-fulfilling prophecy. Merely entertaining the idea that a mental construct is a necessary cause of a behavioral effect, could encourage researchers to ignore evidence that questions this idea. As such, it could slow down the development of theories about alternative causes of the behavioral effect. For instance, it is possible that 
conditioning researchers paid relatively little attention to conditioning via instructions because this effect does not fit well with the idea of the gradual formation of associations in memory as the result of the repeated pairing of stimuli. It is only with the advent of alternative mental construct theories that conditioning via instructions became a viable research topic (e.g., De Houwer, 2009).

Second, when theoretical ideas about the causes of a behavioral proxy do change, this can have disastrous consequences for theories about the mental construct that the proxy was assumed to capture (e.g., association formation, inhibition of representations). When a behavioral effect is treated as a proxy of a mental construct, each instantiation of the behavioral effect by definition becomes a source of information about the mental construct. This implies that theoretical ideas about the mental causes of a behavioral effect (i.e., that the effect cannot be caused by other mental constructs) determine on an a priori basis which findings are considered to be relevant for the development of theories about that mental construct (i.e., all instances of a behavioral effect). A change in ideas about the causes of a behavioral effect would fundamentally alter the set of findings that are deemed to be relevant for theories about a mental construct. Findings that were previously considered to be relevant (i.e., instances of the old behavioral proxy) are no longer relevant whereas findings that were previously considered to be irrelevant are now relevant (i.e., instances of the new behavioral proxy). Such a change would invalidate existing theories and call for new empirical work with new behavioral proxies. For instance, theories about inhibition that were constructed on the basis negative priming data are inaccurate if those data were the result of mental processes other than inhibition. Likewise, if conditioning effects are not necessarily due to the formation of associations in memory, then many existing theories of association formation might have been based on the observation of conditioning effects that were 
actually produced by other mental processes. Hence, these theories would need to be abandoned and new theories would need to be formulated on the basis of new behavioral proxies. Because one cannot foresee future developments in theories about the relation between mental constructs and behavioral effects, there is always the risk that researchers are using the wrong proxies, even when being mindful of the underlying assumptions.

\section{Is the use of proxies actually problematic for the cognitive approach?}

One could still argue that in actual scientific practice, the use of behavioral effects as proxies for mental constructs is not that problematic. In this section, I will discuss three arguments that could lead to this conclusion. First, not all mental constructs are unobservable. For instance, conscious thoughts and feelings are directly observable, be it only by the individual who possesses those thoughts and feelings (e.g., Hayes, 1995; Skinner, 1957). Likewise, identity theorists have put forward the idea that some mental states actually correspond to (physical) neural states and are thus directly observable in principle (e.g., Place, 1956). It is indeed true that the use of proxies becomes unnecessary or less problematic when the to-be-captured mental constructs can be observed directly. Nevertheless, it also seems safe to conclude that many of the mental constructs that are at the core of cognitive psychology (e.g., nodes and associations in semantic networks, schemata, activation and inhibition of representations, attentional filters, storage and retrieval of memory exemplars, short- and long-term memory, working memory, mental models, implicit cognition, unconscious inferences and appraisals, goal-activation, motives, attitudes, etc.) cannot currently be observed directly because they are assumed to be unconscious and thus unavailable to direct observation by the individual or because it is difficult to determine whether or how they relate to directly observable neural states. When studying these unobservable mental constructs, one should be aware of the risks of using behavioral effects 
as proxies.

Second, it could be argued that the use of proxies is unlikely to pose insurmountable problems for cognitive psychology because proxies are used successfully in other sciences. Physicists, for instance, have introduced many constructs that cannot be observed directly (e.g., black holes) and study them by observing other entities that can be observed directly (e.g., the effect that black holes have on the trajectory of other stellar objects). These indirect observations can also be regarded as proxies in that they are used to draw conclusions about an unobservable target based on the direct observation of something other than the target. All conclusions that are based on indirect observations depend on a priori assumptions about the relation between the target and the directly observed entity. In the case of unobservable physical constructs, however, those assumptions are most often grounded in knowledge about physical objects that has been verified independently of the unobservable physical construct (e.g., that all physical objects exert a gravitational pull on each other). In the case of unobservable mental constructs, strong and independent support for a priori assumptions underlying proxies is often lacking. This lack of independent evidence substantially increases the risk of incorrect conclusions when using proxies to study unobservable mental constructs. One could attempt to hedge the risks by using several proxies for each mental construct. Although such an approach is clearly preferable to always using the same proxy of a particular mental construct, there remains a distinct possibility that behavioral effects are never a direct reflection of one particular mental process or mental representation. Moreover, because each behavioral effect is likely to reflect a unique set of mental processes, one can expect that different proxies of the same construct often tend to diverge, raising the question of how to draw conclusions about the mental construct.

Third, one could argue that there is no fundamental difference between direct and 
indirect observations (and thus proxies) in that even conclusions that are based on direct observations are not necessarily correct. It is undoubtedly true that direct observations can be influenced by factors other than the target that is observed (e.g., the genetic make-up of the organism, previous experiences with that target, concepts and hypotheses that were formed in the past). Avoiding the use of proxies of mental constructs will not result in a completely objective study of those constructs. In all likelihood, the aim of complete objectivity can never be reached. Nevertheless, different scientific practices do differ in the probability of being misleading. Drawing conclusions based on indirect observations such as proxies is most often open to more sources of bias than drawing conclusions based on direct observations. Indirect observations not only imply the risks involved with direct observations (because indirect observations of a target are actually direct observations of something else than the target) but also the additional risk of flawed a priori assumptions about the relation between the target and that which is directly observed. Cognitive researchers should thus be aware of the extra risks of using proxies of mental constructs.

\section{The Merits of Combining a Functional and Cognitive Approach}

Even if researchers are aware of the problems associated with the use of behavioral effects as proxies of mental processes, they might continue this practice because they believe that it is the only way to study unobservable mental constructs. The cognitive approach also offers few tools and incentives to always conceptualize behavioral effects without referring to mental constructs. In this section, I propose a functional-cognitive framework that offers an alternative to the use of proxies for mental constructs in cognitive psychology. Within the framework, the cognitive approach is strengthened by grafting it onto a functional approach in which behavioral effects are defined in terms of the causal impact of the environment on behavior. The functional approach not only optimizes the study of mental constructs, it also 
has merit on its own by revealing the environmental causes of behavior. In its turn, cognitive theories about the mental constructs that mediate behavioral effects can lead to the discovery of new functional knowledge. Hence, the functional-cognitive framework highlights the mutually supportive nature of functional and cognitive approaches in psychology.

A cornerstone of the functional approach in psychology is the practice of defining behavioral effects exclusively in terms of elements in the environment (for a detailed discussion, see Chiesa, 1992, 1994; Hayes, 1995; Hayes \& Brownstein, 1986). For instance, classical conditioning effects are not changes in behavior that are due to the formation of associations in memory but changes in behavior that are due to the pairing of stimuli (see De Houwer, 2007, and De Houwer \& Barnes-Holmes, 2010, for a detailed discussion). Likewise, negative priming is not a slowdown in responding that is due to the inhibition of mental representations but a slowdown in responding that is caused by the prior presentation of a related stimulus. These definitions of behavioral effects steer clear of any reference to mental constructs such as association formation or the inhibition of mental representations, including a priori assumptions about the mental causes of the effects (see Table 1).

Combining the cognitive approach in psychology with a functional approach has at least two advantages. First, a functional approach has merit on its own because it provides information about the environmental causes of behavior. Establishing the presence of a behavioral effect implies the identification of the environmental causes of a change in behavior. Note that this goes beyond mere observation in that causal relations cannot be observed directly but need to be inferred. Referring to a change in behavior as a behavioral effect therefore implies a hypothetical explanation of the behavior that is couched in terms of elements in the environment rather than in terms of mental constructs. Although the causal impact of elements in the environment on behavior (i.e., behavioral effects) cannot be 
observed directly, the experimental method provides a solid basis for making causal inferences and thus for establishing the presence of behavioral effects. For instance, claiming that an increase in the time to respond to a target stimulus is an instance of negative priming implies that the increase in response time was caused by the presence of a related prime rather than, for instance, a change in the correct response from one trial to the other. Likewise, claiming that an increase in salivation when hearing a tone is an instance of classical conditioning entails that this change in behavior was caused by the pairing of the tone and food. Experiments can provide the evidence that the pairing was the actual cause of the change in salivation rather than other factors such as the mere presence of food.

A functional approach does not only allow one to explain individual behaviors in terms of elements in the environment, it can also lead to abstract functional knowledge, that is, general knowledge about the environmental determinants of behavior. By studying potential moderators of behavioral effects (e.g., the nature of the stimuli that are presented, the responses that are observed, the organism that is studied, or the situational context in which the effect is observed), more can be learned about the conditions under which certain elements in the environment (e.g., stimulus pairings, the presence of prime stimuli) influence behavior (e.g., produce an increase in salivation or a slowdown in response time). This can lead to functional knowledge that is abstract in the sense that it is assumed to be valid for a large variety of situations. For instance, conditioning research revealed that when reinforcement of a behavior is stopped, this behavior is more likely to persist when it was previously reinforced in an inconsistent manner (i.e., sometimes but not always followed by a reward) than when it was previously reinforced in a consistent manner (i.e., always followed by a reward; e.g., Bouton, 2007). Likewise, negative priming studies support the conclusion that the disruptive impact of a prime on subsequent performance is particularly pronounced 
for those features of the prime that are related to ongoing tasks (e.g., Tipper, Weaver, \& Houghton, 1994). Such abstract functional knowledge allows psychologists to predict and influence individual behaviors inside and outside of the laboratory (e.g., the degree to which behaviors persist after terminating reinforcement or stimuli adversely affect performance).

A second advantage of adopting a functional approach is that it can also increase the success of cognitive psychology. Whereas the functional approach is concerned mainly with the question of when certain elements in the environment influence behavior, the cognitive approach addresses the question of how those elements in the environment influence behavior (also see Bechtel, 2005). It does so by describing the mental processes and mental representations that are assumed to mediate the effect of the environment on behavior. Whereas the description of behavioral effects can provide an explanation of behavior in terms of elements in the environment, the description of mental constructs can provide an explanation of behavioral effects in terms of mental constructs. The functional and cognitive approaches thus focus on two separate levels of explanation that have different explanatory targets (i.e., explanandum) and employ different explanatory constructs (i.e., explanans; see Table 1). Mental explanations are directed at explaining why the environment influences behavior in a certain manner (explanandum) by describing the mental processes and representations that mediate those effects (explanans). As such, the functional approach provides the input on which mental explanations are built. A strong functional approach therefore allows for a strong cognitive approach: The more we know about when a behavioral effect occurs, the more precise we can be about the mental constructs that mediate this effect.

The reverse is also true. Mental explanations should not only provide an account for existing functional knowledge but should also generate new hypotheses about the conditions under which behavioral effects occur. Mental explanations can thus strengthen the functional 
approach by organizing existing functional knowledge (i.e., their heuristic value) and generating new functional knowledge (i.e., their predictive value). For instance, several cognitive models of classical conditioning postulate that associations in memory change only when and to the extent that there is a prediction error, that is, a discrepancy between the stimuli that actually occur and the stimuli that were expected to occur (e.g., Dickinson, 1980). This idea not only allowed these models to account for a variety of known results (e.g., the shape of learning curves, the phenomenon of blocking, see Bouton, 2007) but also led to the discovery of the intuitively implausible fact that additional stimulus pairings can sometimes reduce the size of classical conditioning (i.e., the phenomenon of overexpectation; e.g., Kremer, 1978). In the context of negative priming research, inhibition models not only explained the basic negative priming effect but also led to the discovery that negative priming is particularly pronounced for those stimulus features that are related to ongoing tasks (e.g., Tipper et al., 1994). In sum, the functional and cognitive approaches in psychology are mutually supportive.

Importantly, the interplay between the functional and cognitive approaches works best if both are kept conceptually distinct. By defining behavioral effects strictly in nonmental terms, existing ideas about the mental causes of behavioral effects cannot impose a priori constraints on (a) the different mental explanations of behavioral effects that are being considered or (b) the type of behavioral effects that are deemed to be relevant for theories about a mental construct. The absence of constraints on mental explanations of behavioral effects maximizes the opportunity for theoretical innovation and thus for the discovery of new functional knowledge. For example, the idea that classical conditioning effects can be due not only to association formation but also to the formation and evaluation of propositions (e.g., De Houwer, 2009; Mitchell, De Houwer, \& Lovibond, 2009) has led to a number of 
new discoveries, including the fact that conditioning reflects not only the actual presence of stimuli but also verbal instructions, interventions, and prior knowledge (see De Houwer, 2009, for a review). The absence of a priori constraints on the empirical basis for mental theories, on the other hand, allows one to construct mental explanations without basing them on (potentially incorrect) a priori assumptions. It also makes it possible to spell out the facts that any mental explanation should account for, using terms that do not refer to any specific mental explanation. This in turn allows a continuous and stable development of the functional level of explanation (i.e., the input for mental explanations) even when mental explanations of behavioral effects continue to change.

\section{Potential Arguments against Combining the Functional and Cognitive Approach}

In the previous section, I highlighted the potential benefits of a functional-cognitive framework that combines the strengths of the functional approach with those of the cognitive approach in psychology. It is important to realize that the functional-cognitive framework does not in itself imply a choice for one of both approaches as the most important one. Each approach is based on fundamentally different philosophical assumptions and aims (for a discussion, see Chiesa, 1992, 1994; Gardner, 1987; Hayes, 1995; Hayes \& Brownstein,1986). Which assumptions and aims one chooses to adopt, determines which approach is regarded as most crucial. The main argument of this paper is that, regardless of these assumptions, the functional and cognitive approach can be mutually supportive. For cognitive psychologists, the functional approach can be an instrument for facilitating the discovery of the mental mechanisms that mediate the impact of the environment on behavior. For those who chose to focus on the aims of the functional approach, the cognitive approach can be an instrument for discovering new functional relations between environment and behavior. Both approaches can uphold their fundamentally different philosophical underpinnings and aims while being 
mutually supportive. Moreover, combining the two approaches highlights the fact that each individual study can contribute simultaneously to the aims of both approaches.

It is true, however, that the success of the framework depends on what each of the approaches can add to the other approach. The respective proponents could have doubts about the extent to which other approach can help them achieve their aims. I now address those reservations.

\section{Possible limitations of a functional approach}

Astute readers might have noticed that the functional approach corresponds to the approach that radical behaviorists adopt (e.g., Hayes et al., 2001; Skinner, 1938, 1957). A crucial difference between radical behaviorism and the functional-cognitive framework, however, is that the former excludes whereas the latter promotes interaction between the functional and cognitive approach. Nevertheless, cognitively oriented psychologists might be tempted to resist a functional approach for the same reasons that they rejected radical behaviorism (see Chiesa, 1992, 1994, for an excellent discussion of the core assumptions and critiques of radical behaviorism). Most importantly, cognitive psychologists might argue that certain important topics in psychology are beyond an analysis in terms of effects of the environment on behavior (e.g., Chomsky, 1959, but see Hayes, Barnes-Holmes, \& Roche, 2001). If true, this would potentially limit the importance of the functional approach and suggest that sometimes the cognitive and functional approaches should part ways. There are two ways to respond to this concern. First, given the benefits that a functional approach can have for cognitive psychology, one should do all that is possible to extend the range of the functional approach. The fact that many mental constructs such as “associative link” and “inhibition” cannot be observed directly is unlikely to change. Moreover, there will always be risks when using behavioral effects as proxies for unobservable mental constructs. A 
functional approach provides the best guarantees for a successful cognitive approach and should thus be adopted in combination with the cognitive approach whenever it is possible.

Second, one should not underestimate the power of a functional approach.

Functionally oriented psychologists (e.g., functional contextualists; see Hayes et al., 2001) have continued to refine their functional analyses of all kinds of psychological phenomena such as learning, speaking, remembering, and problem solving. So-called private events (i.e., conscious thoughts and feelings that can be observed directly but only by the person who experiences those events) have also been submitted to detailed functional analyses (see Hayes, 1995, and Hayes \& Brownstein, 1986, for an informative discussion of how private events fit within the functional approach). Instead of clinging to the belief that a functional approach by definition cannot deal with certain types of phenomena, cognitive psychologists would benefit from exploring what has actually been achieved in this respect. Such a dialogue between cognitive psychology and functionally oriented psychologists could lead to many new conceptual tools for describing in non-mental terms the behavioral effects and the conditions under which these effects occur.

One should also be aware of the fact that neuroscience and behavioral genetics can be integrated in a functional approach. For instance, behavioral neuroscientists study the impact of elements in the environment on neural responses such as blood flow in the brain as measured by fMRI (e.g., Carlson, 2010). Likewise, behavioral neuroscientists and behavioral geneticists manipulate neurological and genetic properties of organisms in order to study how this moderates the way in which those organisms respond to elements in the environment. All of this can be achieved without referring to mental constructs. This does not, however, exclude a contribution of neuroscience and genetics to cognitive psychology. On the contrary, by contributing to functional knowledge, they impose important constraints on 
mental explanations and thus help improve and select between different mental explanations.

It should be noted that, also in the context of neuroscience and genetics, the functional approach needs to be kept conceptually separate from the cognitive approach. This implies the view that neural responses (e.g., bloodflow in the amygdala in response to a threatening picture) are not treated as proxies of mental constructs (e.g., processing of threat; see Poldrack, 2006, for a discussion of the problem of inferences about mental processes on the basis of neural data) but simply as effects of elements in the environment on the brain, effects that can be explained by describing mental processes. Such conceptual rigor might require some effort, but produces important benefits in the long run.

\section{Potential risks of a cognitive approach}

Functionally oriented psychologists might also have reasons for resisting a cognitive approach. First, they might have doubts about the ontological status of mental constructs and thus about the feasibility of the aim to uncover the true mental processes that mediate behavioral effects (e.g., Blechtel, 2005; Chiesa, 1994). However, even if one would deny the existence of (unobservable) mental constructs or the ability to uncover the mental constructs that mediate behavioral effects, cognitive theories can still provide useful tools for furthering functional knowledge. The instrumental value of cognitive theories for the functional approach is independent of the ontological status of mental constructs. If those constructs do correspond to a reality, then cognitive theories that closely approximate this reality are likely to be the theories that have the largest heuristic and predictive function. If mental constructs are mere heuristic and predictive tools, than those theories that provide the best tools (i.e., have the largest heuristic and predictive value) will most likely dominate the cognitive approach. Whereas functionally oriented researchers can extend their approach with a cognitive approach based on a purely instrumentalist position, cognitively oriented 
researchers can maintain their realist stance while adopting a functional approach in order to help uncover the nature of the mental world. In sum, the merit of the framework that is proposed in this paper does not depend on ontological assumptions about mental constructs.

Second, functionally oriented researchers might argue that adopting a cognitive approach would detract attention away from the aim of understanding the relation between environment and behavior (e.g., Chiesa, 1994, pp. 152-153). However, it is important to realize that there is no logical reason why a functional approach would be hampered by a cognitive approach in this manner. Mach (1893/1960), who provided much of the philosophical basis for the functional approach in psychology, acknowledged that theories about unobservable mediating processes can have a heuristic and predictive value (see Chiesa, 1994). Radical behaviorists who argued against a cognitive approach (e.g., Skinner, 1937, 1957) did not refute this argument on logical grounds but merely pointed at potential practical problems (i.e., difficulties in studying unobservable constructs). Hence, functionally oriented psychologists are free to explore the benefits of a cognitive approach and engage in a respectful dialogue with cognitive researchers. In sum, once initial preconceptions are set aside, there seems to be little to lose and much to gain from combining the functional and cognitive approaches.

\section{Recommendations for Psychological Research}

The abstract arguments that were developed in this paper do result in concrete recommendations for psychological research in general (see De Houwer \& Barnes-Holmes, 2010, and De Houwer, Gawronski, \& Barnes-Holmes, 2010, for a discussion of the implications of the functional-cognitive framework in learning research and attitude research, respectively). First, behavioral effects and mental constructs need to be separated conceptually. This division means that separate terms should be used for behavioral effects 
(e.g., classical conditioning, negative priming) and mental constructs (e.g., association formation, inhibition). The terms that are used to refer to behavioral effects should avoid references to mental constructs and vice versa. Second, behavioral effects should be defined in terms of the causal impact of specific elements in the (present or past) environment (e.g., the pairing of stimuli, the presence of a certain prime) on specific aspects of behavior (e.g., salivation, the speed of responding to a target). Third, behavioral effects should not be treated as proxies of mental constructs. Negative priming, for instance, is not by definition caused by inhibition and therefore not by definition informative about the properties of inhibition. Fourth, all empirical findings should first be described, as much as possible, in terms of behavioral effects. This recommendation implies that all empirical results should in the first instance be explained at a functional level by describing them as effects of certain stimuli in the (present or past) environment on behavior that occur under certain conditions and that are moderated by certain elements in the environment. These functional explanations should be as general and abstract as possible. Fifth, research should be directed both at developing mental explanations of behavioral effects (i.e., what are the mental processes and representations that mediate these effects) and at improving functional explanations of behavior (what are the abstract elements in the environment that cause and moderate behavior). This recommendation implies that the merits of research should be assessed not only on the basis of the extent to which it advances understanding of mental constructs but also on the basis of how much it contributes to the understanding of the environmental causes of behavior. Following these guidelines can increase the success of both the cognitive approach and the functional approach in psychology and renders these two approaches mutually supportive. 


\section{References}

Bechtel, W. (2005). The challenge of characterizing operations in the mechanisms underlying behavior. Journal of the Experimental Analysis of Behavior, 84, 313-325.

Borsboom, D., Mellenbergh, G. J., \& van Heerden, J. (2004). The concept of validity. Psychological Review, 111, 1061-1071.

Bouton, M. E. (2007). Learning and behavior: A contemporary synthesis. Sunderland, MA: Sinauer Associates, Inc.

Brysbaert, M., \& Rastle, K. (2009). Historical and conceptual issues in psychology. London: Pearson Education.

Carlson, N. R. (2010). Foundations of Behavioral Neuroscience. New York: Pearson.

Chiesa, M. (1992). Radical behaviorism and scientific frameworks: From mechanistic to relational accounts. American Psychologist, 47, 1287-1299.

Chiesa, M. (1994). Radical behaviorism: The philosophy and the science. Boston, MA: Authors' Cooperative.

Chomsky, N. (1959). A Review of “Verbal Behavior” by B. F. Skinner. Language, 35, 26-58.

De Houwer, J. (2007). A conceptual and theoretical analysis of evaluative conditioning. The Spanish Journal of Psychology, 10, 230-241.

De Houwer, J. (2009). What are association formation models good for? Learning \& Behavior, 37, 25-27.

De Houwer, J., \& Barnes-Holmes, D. (2010). A general framework for learning research. Manuscript in preparation.

De Houwer, J., Gawronski, B., \& Barnes-Holmes, D. (2010). A behavioral-cognitive framework for attitude research. Manuscript in preparation.

Dickinson, A. (1980). Contemporary Animal Learning Theory. Cambridge: Cambridge 
University Press.

Gardner, H. (1987). The mind's new science: A history of the cognitive revolution. New York: Basic Books.

Hayes, S. C. (1995). Why cognitions are not causes. The Behavior Therapist, 18, 59-60.

Hayes, S. C., Barnes-Holmes, D., \& Roche, B. (Eds.). (2001). Relational Frame Theory: A Post-Skinnerian account of human language and cognition. New York: Plenum Press.

Hayes, S. C., \& Brownstein, A. J. (1986). Mentalism, behavior-behavior relations, and a behavior-analytic view of the purposes of science. The Behavior Analyst, 9, 175-190.

Hempel, C. G. (1970). Aspects of scientific explanation and other essays in the philosophy of science. New York: Free Press.

Joormann, J. (2004). Attentional bias in dysphoria: The role of inhibitory processes. Cognition and Emotion, 18, 125-147.

Kremer, E. F. (1978). The Rescorla-Wagner model: Losses in associative strength in compound conditioned stimuli. Journal of Experimental Psychology: Animal Behavior Processes, 4, 22-36.

Lamberts, K., \& Goldstone, R. (2004). Handbook of cognition. London: Sage Publications.

Mach, E. (1893/1960). The science of mechanics: A critical and historical account of its development. Illinois: Open Court.

Mitchell, C. J., De Houwer, J., \& Lovibond, P. F. (2009). The propositional nature of human associative learning. Behavioral and Brain Sciences, 32, 183-198.

Neill, W. T., \& Valdes, L. A. (1992). The persistence of negative priming: Steady-state or decay? Journal of Experimental Psychology: Learning, Memory, \& Cognition, 18, 565-576.

Neisser, U. (1967). Cognitive psychology. Englewood Cliffs, NJ: Prentice-Hall. 
Place, U. T. (1956). Is consciousness a brain process? British Journal of Psychology, 47, p. $44-50$

Poldrack, R. A. (2006). Can cognitive processes be inferred from neuroimaging data? Trends in Cognitive Sciences, 10, 59-63.

Skinner, B.F. (1938). The Behavior of Organisms: An Experimental Analysis. New York: Appleton-Century.

Skinner, B. F. (1957). Verbal Behavior. Englewood Cliffs, NJ: Prentice-Hall.

Tipper, S. P. (1985). The negative priming effect: Inhibitory priming by ignored objects. Quarterly Journal of Experimental Psychology, 37A, 571-590.

Tipper, S. P., Weaver, B., \& Houghton, G. (1994). Behavioural goals determine inhibitory mechanisms of selective attention. Quarterly Journal of Experimental Psychology, 47A, 809-840. 


\section{Author Note}

Jan De Houwer, Ghent University, Ghent, Belgium. The preparation of this paper was made possible by Grants BOF/GOA2006/001 and BOF09/01M00209 of Ghent University and by Travel Grant K142406N01 of the Research Foundation-Flanders (FWO-Vlaanderen, Belgium). I would like to thank my mentor Paul Eelen for providing the starting point for the ideas expressed in this paper and the many people with whom I discussed these ideas over the past five years, most prominently Dermot Barnes-Holmes, Bertram Gawronski, and Agnes Moors. Thanks also to Dermot Barnes-Holmes, Marc Brysbaert, Peter Lovibond, Leonel Garcia-Marques, and Tom Verguts for comments on earlier drafts. Correspondence should be addressed to Jan De Houwer, Ghent University, Henri Dunantlaan 2, B-9000 Ghent, Belgium. Electronic mail can be sent to Jan.DeHouwer@UGent.be . 
Table 1. Core aspects of the functional and cognitive approaches to classical conditioning and negative priming.

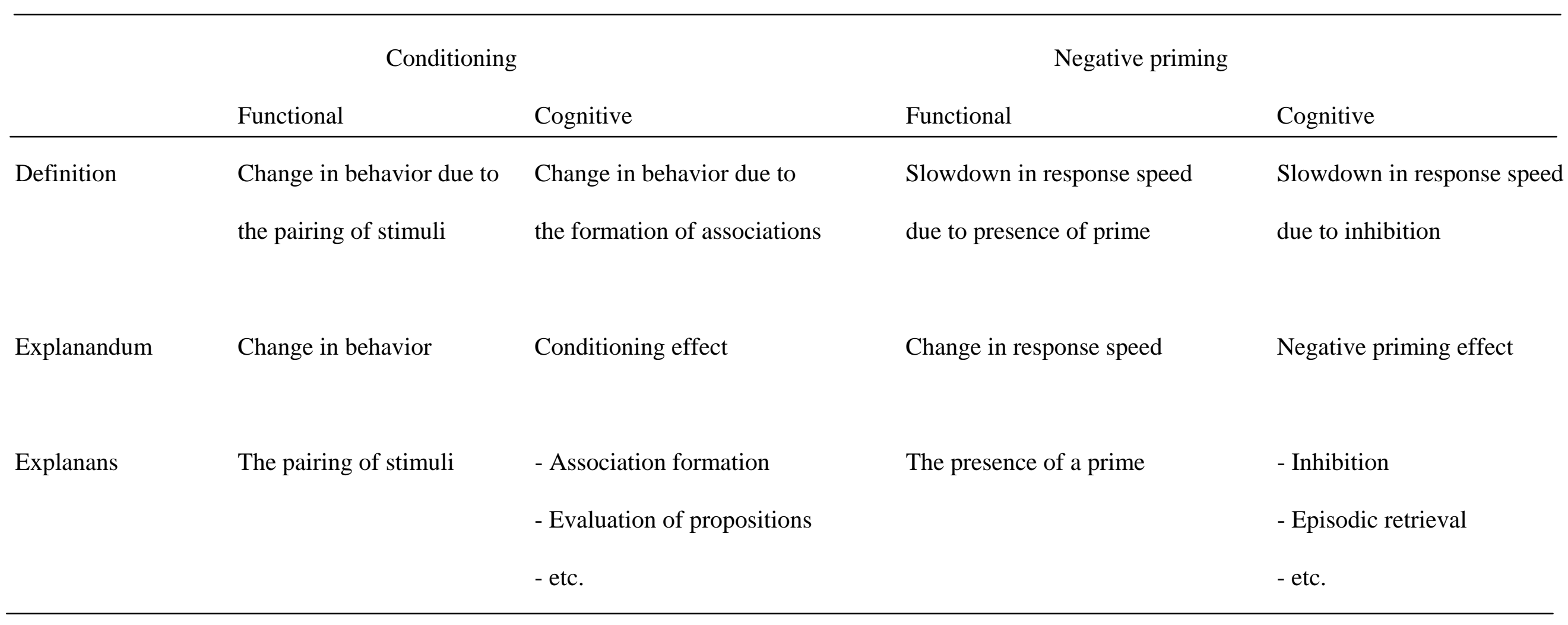

